\title{
THE MOLECULAR PHYLOGENY OF THE Q$_{9}$-EQUIPPED ASCOMYCETOUS TELEOMORPHIC YEAST GENUS DEBAR YOMYCES LODDER ET KREGER-VAN RIJ BASED ON THE PARTIAL SEQUENCES OF 18S AND 26S RIBOSOMAL RIBONUCLEIC ACIDS ${ }^{1}$
}

\author{
YUZO YAMADA, ${ }^{2}$ TAKAHIKO NAGAHAMA, AND ISAO BANNO* \\ Laboratory of Applied Microbiology, Department of Agricultural Chemistry, \\ Shizuoka University, Shizuoka 422, Japan \\ *Institute for Fermentation, Osaka, Jusohon-machi, \\ Yodogawa-ku, Osaka 532, Japan \\ (Received April 26, 1991)
}

The partial base sequences of $18 \mathrm{~S}$ and $26 \mathrm{~S}$ rRNAs were examined in eighteen strains of Debaryomyces, Torulaspora, and Yamadazyma species including two strains of D. udenii. All of the strains of Debaryomyces species constituted a single group (cluster) phylogenetically. In the partial base sequence (positions 493 through 622, 130 bases) of $26 \mathrm{~S}$ rRNA, the maximum homologies were 79-99\% among Debaryomyces species. T. globosa and $Y$. philogaea had $71-78 \%$ and $81-87 \%$ maximum homologies, respectively, with Debaryomyces species. In the partial base sequence (positions 1611 through 1835, 225 bases) of 26S rRNA, the base differences numbered 5-0 among Debaryomyces species. T. globosa and $Y$. philogaea had $15-13$ and 10-8 base differences, respectively, with $D e-$ baryomyces species. In the partial base sequence (positions 1451 through 1618, 168 bases) of $18 \mathrm{~S}$ rRNA, Debaryomyces species were divided into two subgroups (subclusters). The first subgroup was comprised of $D$. hansenii, $D$. melissophilus, $D$. udenii, and so on, and the second subgroup comprised of $D$. castellii, $D$. polymorphus, $D$. yamadae, and so on. The base difference numbered 1 between the two subgroups. T. globosa and $Y$. philogaea had 5-4 and 1-0 base differences, respectively, with Debaryo-

1 This constitutes Part XLII of a series entitled "Significance of the coenzyme Q system in the classification of yeasts and yeast-like organisms." For Part XLI, see ref. 21.

${ }^{2}$ Address reprint requests to: Dr. Yuzo Yamada, Laboratory of Applied Microbiology, Department of Agricultural Chemistry, Shizuoka University, 836 Ohya, Shizuoka 422, Japan.

Abbreviations: Co-Q, coenzyme Q or ubiquinone; Q-9 or $\mathrm{Q}_{9}$ and Q-6 or $\mathrm{Q}_{6}$, coenzyme Q or ubiquinone homologues with 9 and 6 isoprene units, respectively, in a side chain; rRNA, ribosomal RNA; S, Svedberg value. 
myces species. Between T. globosa and S. cerevisiae, there was 1 base difference. D. tamarii occupied a distant position (maximum homologies, 63-71\%; base differences, 50-48 and 20-19, respectively).

The genus Debaryomyces Lodder et Kreger-van Rij was established with the type species, Debaryomyces hansenii (Zopf) Lodder et Kreger-van Rij (6-8, 11,1315). This genus is characterized morphologically by the formation of warty (or verrucate) ascospores $(7,8,11)$, physiologically by the slow, weak but not vigorous fermentation $(6,7,11,14,17)$, and chemotaxonomically by the Q-9 system $(1,13$, $17,20,22,23)$.

A new, soil-associated species was recently described in the genus Debaryomyces as Debaryomyces udenii van der Walt, Smith et Yamada (18). However, this species is recognized to be an intermediate between $P$. abadiae, $P$. carsonii, and $P$. etchellsii which form more or less glabrous ascospores and the presently accepted Debaryomyces species which form verrucate ascospores, since it produces colliculate to pusticulate rather than verrucate ascospores (18).

This paper is concerned with the partial base sequences of $18 \mathrm{~S}$ and 26S rRNAs of $D$. udenii along with the presently accepted species of the genus Debaryomyces from the phylogenetic point of view.

\section{MATERIALS AND METHODS}

Yeast strains examined and cultivation. The yeast strains examined are listed in Table 1. The eighteen strains were grown as described previously (19).

Preparation of rRNAs. The rRNAs of the yeast strains were prepared as described previously (19).

Partial base sequencing of $18 S$ and $26 S$ rRNAs. The partial base sequences of $18 \mathrm{~S}$ and $26 \mathrm{~S}$ rRNAs of the yeast strains were determined by the method of Lane et al. (10) using reverse transcriptase. The oligonucleotide DNA primers used in this experiment were 5'-ACGGGCGGTGTGTAC-3', which is complementary to the sequence of positions 1641 through 1627 of 18S rRNA (in Saccharomyces cerevisiae) (12) and 5'-GGTCCGTGTTTCAAGACGG-3' and 5'-TTGGAGACCTGCTGCGG-3', which are complementary to the sequences of positions 654 through 636 and 1857 through 1841, respectively, of 26S rRNA (in S. cerevisiae) (4).

Chemicals. The chemicals used in this experiment were the same as those described previously (19).

\section{RESULTS}

Partial base sequences in positions 493 through 622 of $26 S$ rRNA

The primary partial base sequences of the strains examined in Debaryomyces, Torulaspora, and Yamadazyma species were aligned (Fig. 1). The maximum 
Table 1. The strains examined of Debaryomyces, Torulaspora, and Yamadazyma species.

\begin{tabular}{|c|c|c|}
\hline Species and strain & Other designation & Co-Q system ${ }^{a}$ \\
\hline \multicolumn{3}{|c|}{ Debaryomyces Lodder et Kreger-van Rij } \\
\hline \multicolumn{3}{|l|}{ D. castellii Capriotti } \\
\hline IFO $1359^{*}$ & CBS 2923, JCM 6177, A. Capriotti & Q-9 \\
\hline \multicolumn{3}{|l|}{ D. coudertii Saez } \\
\hline JCM $2387^{*}$ & CBS 5167 , IFO 1817 , H. Saez & Q-9 \\
\hline \multicolumn{3}{|c|}{ D. hansenii (Zopf) Lodder et Kreger-van Rij var. hansenii } \\
\hline IFO $0083^{*}$ & CBS 767, JCM 1990 & Q-9 \\
\hline \multicolumn{3}{|c|}{ D. hansenii (Zopf) Lodder et Kreger-van Rij var. fabryi (Ota) } \\
\hline \multicolumn{3}{|l|}{ Nakase et Suzuki } \\
\hline IFO $0015^{*}$ & CBS 789, JCM 2104, M. Ota & Q-9 \\
\hline \multicolumn{3}{|l|}{ D. marama di Menna } \\
\hline IFO $0668^{*}$ & CBS 1958, JCM 1528, M. E. di Menna & Q-9 \\
\hline \multicolumn{3}{|c|}{ M. Mikata } \\
\hline \multicolumn{3}{|c|}{ D. melissophilus (van der Walt et van der Klift) Kurtzman et Kreger-van Rij } \\
\hline IFO $1900^{*}$ & CBS 6344, JCM 1707, J. P. v.d. Walt & Q-9 \\
\hline \multicolumn{3}{|c|}{ D. nepalensis Goto et Sugiyama } \\
\hline JCM 2095* & AJ 4967, CBS 5921, IFO 1428, J. Sugiyama & Q-9 \\
\hline \multicolumn{3}{|c|}{ D. polymorphus (Klöcker) Price et Phaff } \\
\hline IFO $1166^{*}$ & CBS 186 , JCM 3647 , O. Winge & Q-9 \\
\hline \multicolumn{3}{|c|}{ D. pseudopolymorphus (Ramirez et Boidin) Price et Phaff } \\
\hline IFO $1026^{*}$ & CBS 2008, JCM 3652, J. Boidin & Q-9 \\
\hline \multicolumn{3}{|c|}{ D. tamarii Ohara et Nonomura } \\
\hline IFO 0854* & CBS 4333, JCM 6351, Y. Ohara & Q-9 \\
\hline \multicolumn{3}{|c|}{ D. udenii van der Walt, Smith et Yamada } \\
\hline JCM 7855* & CBS 7056, J. P. v.d. Walt & Q-9 \\
\hline JCM 7856 & CBS 7057 , J. P. v.d. Walt & \\
\hline $\begin{array}{l}\text { D. vanrijiae (van der V } \\
\text { IFO 0934* }\end{array}$ & D. vanrijiae (van der Walt et Tscheuschner) Abadie, Pignal et Jacob var. vanrijiae & Q-9 \\
\hline \multicolumn{3}{|c|}{$\begin{array}{l}\text { D. vanrijiae (van der Walt et Tscheuschner) Abadie, Pignal et Jacob var. yarrowii } \\
\text { (Santa Maria et Garcia-Aser) Kreger-van Rij }\end{array}$} \\
\hline IFO $1818^{*}$ & CBS 6246, JCM 6190, J. Santa Maria & Q-9 \\
\hline \multicolumn{3}{|c|}{ D. yamadae (van der Walt et Johannsen) van der Walt, Smith et Yamada } \\
\hline JCM $6191^{*}$ & CBS 7036, J. P. v.d. Walt & Q-9 \\
\hline \multicolumn{3}{|l|}{ Torulaspora Lindner } \\
\hline \multicolumn{3}{|c|}{ T. globosa (Klöcker) van der Walt et Johannsen } \\
\hline IFO $1160^{*}$ & CBS 764 & Q-6 \\
\hline \multicolumn{3}{|c|}{ Yamadazyma Billon-Grand } \\
\hline \multicolumn{3}{|c|}{ Y. philogaea (van der Walt et Johannsen) Billon-Grand } \\
\hline CBS $6696^{*}$ & & Q-9 \\
\hline
\end{tabular}

a Data from Yamada and Kondo (20), Yamada et al. $(22,23)$, Nakase and Suzuki (13), van der Walt and Johannsen (17), van der Walt et al. (18), and Billon-Grand $(1,2)$.

* Type strain.

Abbreviations: IFO, Institute for Fermentation, Osaka, Osaka, Japan; CBS, Centraalbureau voor Schimmelcultures, Delft, The Netherlands; JCM, Japan Collection of Microorganisms, Institute of Physical and Chemical Research, Wako, Saitama, Japan.

homology (\%) was calculated in the respective pairs of the strains by computer analysis using a Hitachi DNAsis (Ver. 7, Hitachi Software Engineering Co., Ltd., Tokyo, Japan) instead of calculating the number of base differences, since the base 
substitutions frequently occurred in this region.

As shown in Fig. 2, the maximum homologies were 79-99\% among the strains of the species of the genus Debaryomyces, except for D. tamarii IFO 0854 which had abnormally low maximum homologies $(63-71 \%)$ with the respective species of the genus Debaryomyces. The maximum homologies of $T$. globosa IFO 1160 were 71$78 \%$ and $87 \%$ with the strains of Debaryomyces species and S. cerevisiae, respectively. Yamadazyma philogaea CBS 6696 had $81-87 \%$ and $78 \%$ maximum homologies with the strains of Debaryomyces species and $T$. globosa, respectively.

Based on the calculated maximum homologies, a dendrogram was drawn by the simple linkage method (5). As shown in Fig. 3, the strains of Debaryomyces species constituted a single cluster with $86-99 \%$ maximum homologies. Yamada-

1 D.castellii IFO 1359*

2 D.coudertii JCH 2387*

3 D.hansenii var.hansenii IF0 $0083 *$

4 D.hansenii var fabryi IFO 0015*

5 D. narama IFO 0668*

6 IFO 1878

7 D.melissophilus IF0 1900*

8 D.nepalensis JCH 2085*

9 D.polynorphus IPO 1166 *

10 D.pseudopolyeorphus IP0 1026*

11 D.tanarii IPO 0854 *

12 D.udenii JCM 7855*

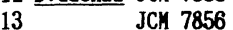

14 D.vanri.jiae var.vanrijiae IFO $0934 *$

15 D.vanri.jiae var.yarrowii IF0 1818*

16 D.yanadae JCH $6191 *$

17 T.globosa IF0 $1160 *$

18 Y.philogaea CBS $6696 *$

19 S.cerevisiae IFO 2376 $\begin{array}{llllll}.483 & .501 & .511 & .521 & .531 & .541\end{array}$

GGCCAGCA UCGGUUUGGA UGGUGGGAUA AUGACUUGG AAUUG.CUNU GCUUCGGU.NA GECCAGCA UCGgUUUGGA UGGUAGGAUA AUGAUUAAG AAUGUGGCUCU GCUUCGGUGG gGCCAGCA UCGgUUUGGA UGgUAGgAUA AUGACUAAGg AAUGUGGCUCU ACUUCGgUGGA GGCCAGCA UCGGUUUGGA UGGUAGGAS AUGACUAAGG AAUGUEGCUCU ACUUCGGUGGA GGCCAGCA UCGGUUUGG CGGUAGGAUA AUGAUUAAG AAUGUGCCUCU GCEGCGGUGA GGCCAGCA UCGGUUUGGA CGGUAGGAUA AUGAUUAAGG AAUGUGGCUCU ACUUCGGUGGA GGCCAGCA UCAAUUUGGA UGAUGGAUA AUGAAUCAGG AAUGUAGCUUU ACUUCGGUGAA GGCCAGCA UCGGUUUGGA UGGUAGGAUA AUGAUUAAGG AAUGUGGCUCU ACUUCGGUGGA GGCCAGCA UCGGUUUGGA UGGUAGGAUA AUGACUUGG AAUGUGACUUU ACUUCGGUAAA GGCCAGCA UCGGUUUGGA UGGUAGGAUA AUGACUUGGG AAUGUGGCUUU GCUUCGGUAAA GGCUAACA UCAGUUUUGC CGGAUGGAUA A..A.U.GGU AAAGUGGCAUU ..UUCGG.AU. GGCCAGCA UCGGUUUGGA UGGUAGGAUA AUGAUUAAGG A.UGUGAGCGC ..UUCGGUNU. GGCCAGCA UCGGUUUGGA UGGUAGGAUA AUGAUUAAGG AAUGUG.GCGC .. UUCGGUNU.

gGCCAGCA UCGgUUUGGa UGgUAGgAUA AUGUCUUGgG AAUGUGGCUUU GCUUCGgUAAA GGCCAGCA UCGGUUUGGA UGGUAGGAUA AUGACUUGG AAUGUGGCUUU GCUUCGGUAAA GGCCAGCA UCGGUUUGGA UGGUGGGAUA AUGACAUUGG AAUGUGGCUCU GCUUCGGUAGA GGCCAGCA UCAGUUUUGG CGGUAGGAUA AAUCUGCAG. AAUGUAGCUU. GCUUCGGC.AA GGCCAGCA UCAGUUUGGA CGGUGGGAUA AUAGCGGAGG AAUGUGGCUCG GCUUCGGUNGA GGCCAGCA UCAGUUUUGG UGGCAGGAUA AAUCCAUAGG AAUGUAGCUU. GCCUCGGU.AA

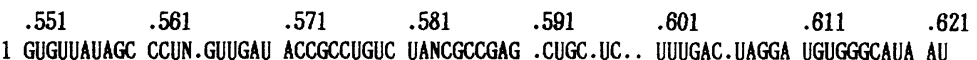

2 GUGUUAUAGC CUUG.GUUGAU ACUGCCUGUC UAGAGACGAG . CUGCGUC.. UUUGAC. UAGGA UGUUGGCAUA AU

3 GUGUUAUAGC CUNN.GUUGAU ACUGCCNGUC UAGACCGAGG .CUGCGUC. $U$ UUUGAC. UAGGA UGUUGGCAUA AU

4 gUGUUAUAGC CUUN.GUUGAU ACUGCCUGUC UAGACCGAGg .CAGCGUC.. UUUGAC. UAGGA UGCUGGCAUA AU

5 GUGUUAUAGC CUUN.GUUGAU GCUGCCUGUC UAGACCGAGG ACUGCGUV.U UUUGAC. UAGGA UCUUGGCAUA AU

6 GUGUUAUAGC CUUN.GUUGAU GCUGCCUGUC UAGACCGAGG ACUGCGUC.U UUUGAC. UAGGA UGCUGGCAUA AU

7 GUGUUAUAGC CUGU.GUUGAU ACCGUCUAUC UAGAUUGAGG ACUGCGUC.U UUUGAC. AAGGA UGUUGGCAUA AU

8 GUGUUAUAGC CUUG. GUUGAU ACUGCCUGUC UAGAN.GCGA GCUGCGUN.. UUUGAC. UAGGA UGCUGGCAUA AU

9 GUGUUAUAGC CCUN.GUUGAU ACUGCCUGUC UAGACCGAGG ACUGCGUC.. UUUGAC. UAGGA UGCUGGCAUA AU

10 GUGUUAUAGC CCUN.GUUGAU ACUACCUGUC UAGACCGAGG ACUGCGUC.. UUUGAC. UAGGA UGCUGGCAUA AU

11 GUGUUAUAGC UUUAUCGAAAU ACGUAACGGC GGGAUUGAGG ACCGCG.C.U UUAUGC. AAGGA UGUUGGCGUA AU

12 GUGUUAUAGC CUUA.GUUGAU ACUGCCUGUC UAGACCGACG .CUGC. UC.. UUUGAC. UAGGA UGUUGGCAUA AU

13 NUGUUAUAGC CUUA.GUUGAU ACUGCCUGUC UAGACCGA.G .CUGC.UC.. UUUGAC. UAGGA UGUUGGCAUA AU

14 GUGUUAUAGC CCUU.GUUGAU GCUACCUGUC UAGACCGAGG ACUGCGUC.. UUUGAC. UAGGA UGUUGGCAUA AU

15 GUGUUAUAGC GCUN.GUUGAU GCUACCUGUC UGAGCCGAGG ACUGCGUC.. UUUUGAC. UAGGA UGUUGGCAUA AU

16 GUGUUAUAG. GCUUUGUUGAU ACCACCUGUC UAGACCGUGG .CUGCGUC.. UUUGACGUAGGA UGUUGGCAUA AU

17 GUGUUAUAGC CUGU. UGAAAU ACUGCCAGCC GGGACUGAGG ACUGCGACUU UUAGUC. AAGGA UGCUGGCAUA AU

18 GUGUUAUAGC CUNG.GUUGAU ACCGCCUGUC UAGAGCUGAG GCUGCGUCUU UGAGU... AGGA UGCUGGCAUA AU

19 GUAUUAUAGC CUGU.GGGAAU ACUGCCAGCU GGGACUGAGG ACUGCGACGU A.AGUC. AAGGA UGCUGGCAUA AU

Fig. 1. The primary partial base sequences in positions 493 through 622 of $26 \mathrm{~S}$ rRNA in strains of Debaryomyces, Torulaspora, and Yamadazyma species. The primary partial sequences were aligned. The arabic numerals indicate positions in $S$. cerevisiae (4). N, A, G, C, or U. * Type strain. 
zyma philogaea CBS 6696 was linked to the cluster of Debaryomyces species at $87 \%$ maximum homology. Torulaspora globosa IFO 1160 and S. cerevisiae IFO 2376 constituted another cluster with $87 \%$ maximum homology. These two clusters were linked to each other at $78 \%$ maximum homology. The linkage of $D$. tamarii IFO 0854 was at $76 \%$ maximum homology.

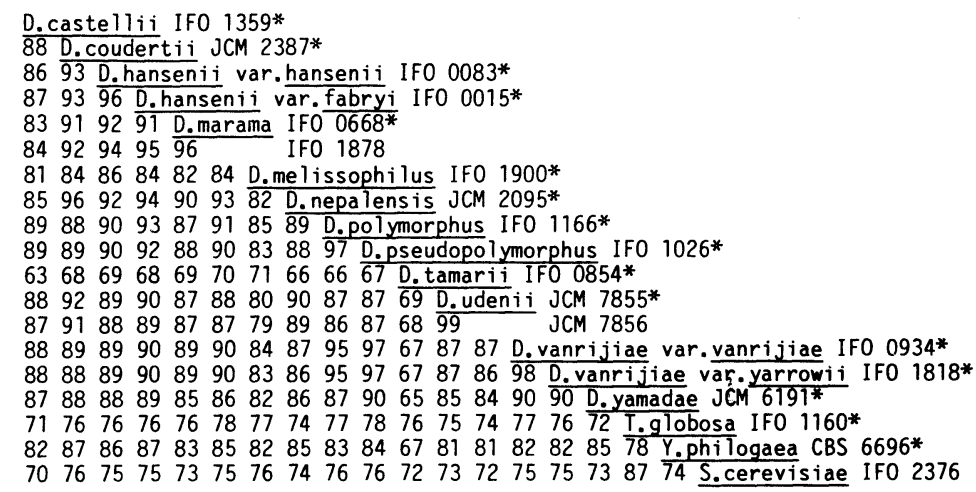

Fig. 2. A triangle matrix based on the calculated maximum homologies in the partial base sequences in positions 493 through 622 of 26S rRNA in strains of Debaryomyces, Torulaspora, and Yamadazyma species. The maximum homologies (\%) were calculated by computer analysis using a Hitachi DNAsis in positions 493 through 622 (130 bases) of 26S rRNA. * Type strain.

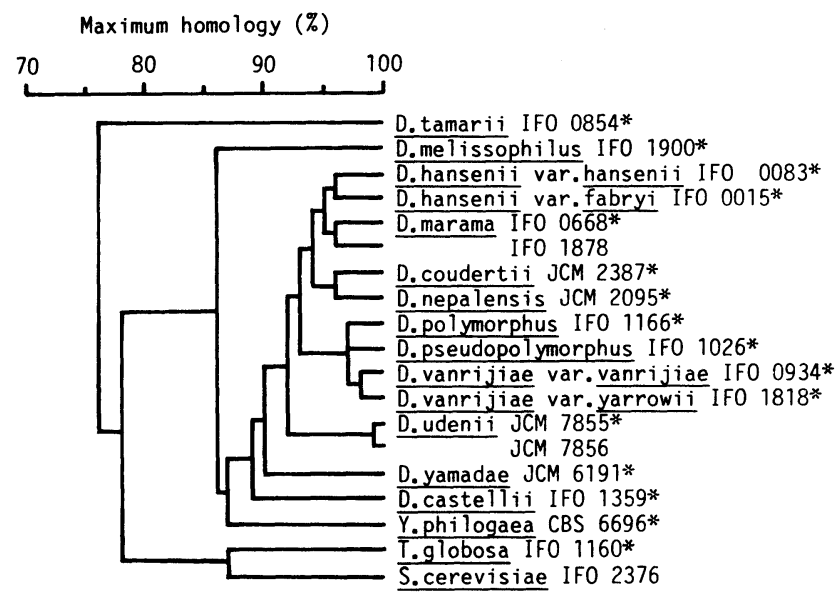

Fig. 3. A dendrogram based on the calculated maximum homologies in the partial base sequences in positions 493 through 622 of $26 \mathrm{~S}$ rRNA in strains of Debaryomyces, Torulaspora, and Yamadazyma species. The dendrogram was drawn by the simple linkage method (5). * Type strain. 
Partial base sequences in positions 1611 through 1835 of $26 \mathrm{~S} r R N A$

The primary partial base sequences of the strains of Debaryomyces, Torulaspora, and Yamadazyma species were aligned (Fig. 4). The number of base differences was calculated among the strains examined.

As shown in Fig. 5, the base differences numbered 5-0 among the strains of Debaryomyces species except for $D$. tamarii IFO 0854. There was no base difference among the strains of $D$. hansenii var. hansenii, $D$. hansenii var. fabryi, $D$. marama, and so on, and of $D$. polymorphus, $D$. pseudopolymorphus, $D$. vanrijiae var. vanrijiae, and $D$. yamadae, and between the strains of $D$. udenii, respectively. Debaryomyces melissophilus IFO 1900 had 5-2 base differences, compared with the strains of other Debaryomyces species. The number of base differences was very large in $D$. tamarii IFO 0854 (50-48). Torulaspora globosa IFO 1160 and $Y$. philogaea CBS 6696 had 15-13 and 10-8 base differences, respectively, from the strains of Debaryomyces species.

Based on the calculated number of base differences, a dendrogram was drawn by the simple linkage method (5). As shown in Fig. 6, all the strains of Debaryomyces species constituted a single cluster within 2 base differences. Yamadazyma philogaea CBS 6696 was linked to the cluster of Debaryomyces species at 8 base differences. Torulaspora globosa IFO 1160 and $S$. cerevisiae IFO 2376 constituted a

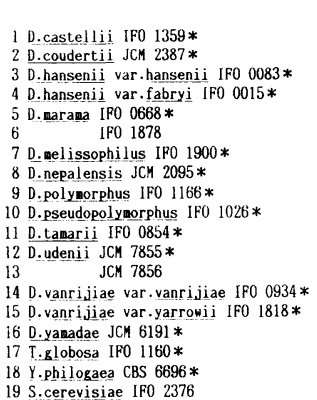
ae IFO 2376

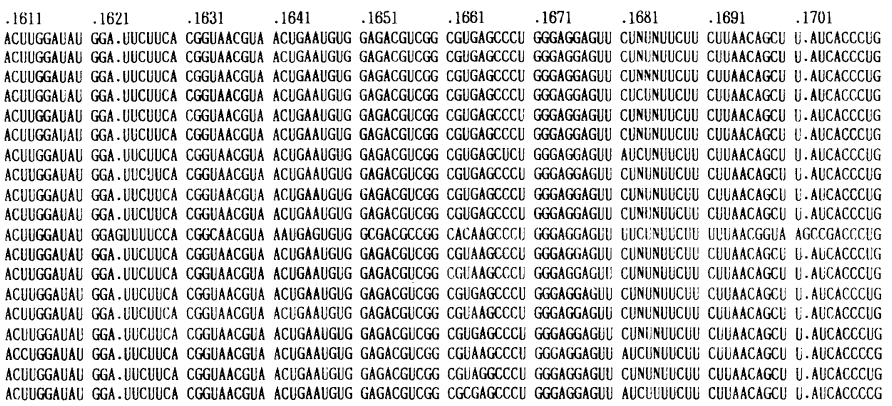

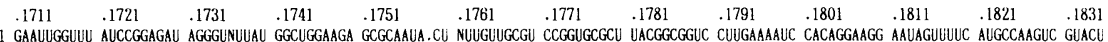

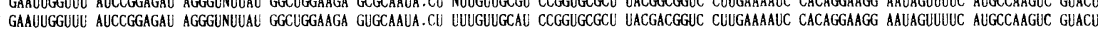
3 GAALUGGUUU AUCCGGAGAU AGGGUNUUAU GGCUNGAAGA GUGCAAUA.CU NUGULGCAU CCGGUGCGCU UACGACGGUC CUUGAAAAUC CACAGGAGG AAUGGUUUC AUGCCAAGUC GUACU 5 GAAUUGGLU AUCCGGAGAU AGGGUNUUAU GGCUGGAAGA GLGCAAUA.CU UUUGUUGCAU CCGGUGCGCU UACGACGGUC CUUGAAAAUC CACAGGAAGG AAUAGOUUUC AUGCCAAGUC GUACD 5 GAALUGGUUU AUCCGGAGAU AGGGUNUUAU GGCUGGAAGA GUGCAAUA.CU UUUGUUGCAU CCGGUGCGCU UACGACGGUC CUUGAAAAUC CACAGGAAGG AAUAGUUULC AUGCCAAGUC GUACL 6 GAALUGGUUU AUCCGGAGAU AGGGUNUUAU GGCLGGAAGA GUGCAAUA.CU UUUGUUGCAU CCGGUGCGCU UACGACGGUC CUUGAAAAUC CACAGGAAGG AAUAGUUUUC AUGCCAAGUC GUACL 7 GAALUGGUUU AUCCGGAGAU AGGGUNUUAU GGCUGGAGA GGGCAAUA.CU UUUGUUGCGU CGGGUGCGCU UACGACGGUC CUUGAAAAUC CACAGGAAGG AAUAGUULUC AUGCCAAGUC GUACU 8 GAAUUGGUUU AUCCGGAGAU AGGGUCEUAU GGCUGGAAGA GUGCAALA.CU UUUGUUGCNU CCGGUGCGCU UACGACGGUC CUUGAAAAUC CACAGGAAGG AAUAGLLUUC AUGCCAAGIC GUACU 9 GAAUUGGUUU AUCCGGAGAU AGGGUNUUAU GGCUGGAAGA GCGCAAUA.CU UUUGGUGCGU CCGGUGCGCU UACGACGGUC CUUGAAAALC CACAGGAAGG AAUAGUUUUC AUGCCAAGUC GUACU 10 GAALUGGUUU AUCCGGAGAU AGGGUNUUAU GGCUGGAAGA GCGCAAUA.CU UUUGUUGCGU CCGGUGCGCU UACGACGGUC CUUGAAAAUC CACAGGAAGG AAUAGUUUUCC AUGCCAAGUC GUACU 11 GAAUUGGUUU AGCCAGCGGU AGGGUNUGUU UACCGGAAGA GCCCGACAUCU ULCGUCGGUU CCGGUGCGCU UGUGACGGUC CUUGAAAAGC CGCAUGAAGU UUUUGCLUUC AUACCAAGUC GLACL 12 GAAUUGGUUU AUCCGGAGAL AGGGUNULAU GGCUGGAAGA GUGCAAUA.CU UUUGUUGCAU CCGGUGCGCU UACGACGGUC CUUGAAAAUC CACAGGAAGG AAUAGUUUUC AUGCCAAGUC GUACU 13 GAAUUGGUU AUCCGGAGAU AGGGUNUUAU GGCUGGAAGA GUGCAALA.CU UUUGLUGCAU CCGGUGCGCU UACGACGGUC CUUGAAAAUC CACAGGAAGG AAUAGUUUUC AUGCCAAGLC GUACU 14 GAALUGGUUU AUCCGGAGAU AGGGUNUUAU GGCLGGAAGA GCGCAAUA.CU UUUGUUGCGU CCGGUGCGCU UACGACGGUC CUUGAAAAUC CACAGGAAGG AAUAGUUUUC AUGCCAAGUC GUACU 15 GAAUUGGUU AUCCGGGGAU AGGGUNUUAU GGCUGGAGA GCGCAAUA.CU UUUGUUGCUU CCGGUGCGCU UACGACGGAC CUUGAAAAUC CACAGGAAGG AAUAGUUUUC AUGCCAAGIC GUACU 16 GAAUUGGUU AUCCGGAGU AGGOUNUAU GGCUGGAAGA GCGCAAUA.CU UUUGUUCGU CCGGUGCGCU UACGACGGUC CUUGAAAAUC CACAGGAAGG AAUAGUUUUC AUGCCAAGUC GUACL 17 GAAUUGGUUU AUCCGGGGAU GGGGUNUUAU GGCUGGAAGA GCGCAGCA. UC UUUGCUGGCU CCGGLGCGCU UACGACGGCC CUUGAAAAUC CACAGGAAGG AAUAGUUUUC AUGCCAGGUC GLACU 18 GAAUUGGUUU AUCCGGAGAU AGGGUNUUAU GGCUGGAGGA GCGCAGUA.CL LUUGCU.CGU CUGGCGCGCC UACGACGGUC CUUGAAAAUC CACAGGAGGG AAUAGUUUUC AUGCCAAGLC GUACU

Fig. 4. The primary partial base sequences in positions 1611 through 1835 of $26 \mathrm{~S}$ rRNA in strains of Debaryomyces, Torulaspora, and Yamadazyma species. The primary partial sequences were aligned. The arabic numerals indicate positions in $S$. cerevisiae (4). N, A, G, C, or U. * Type strain. 


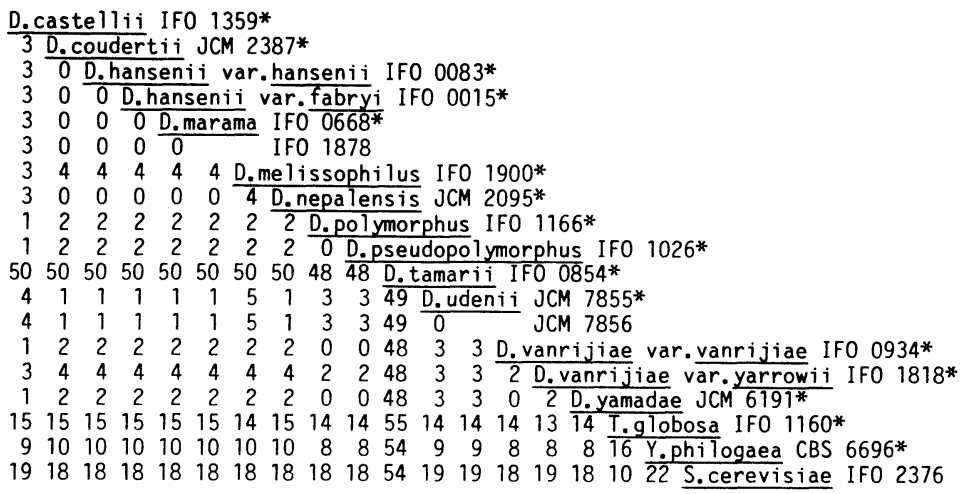

Fig. 5. A triangle matrix based on the calculated number of base differences in the partial base sequences in positions 1611 through 1835 of 26S rRNA in strains of Debaryomyces, Torulaspora, and Yamadazyma species. The number of base differences was calculated in positions 1611 through 1835 (225 bases) of 26S rRNA. * Type strain.

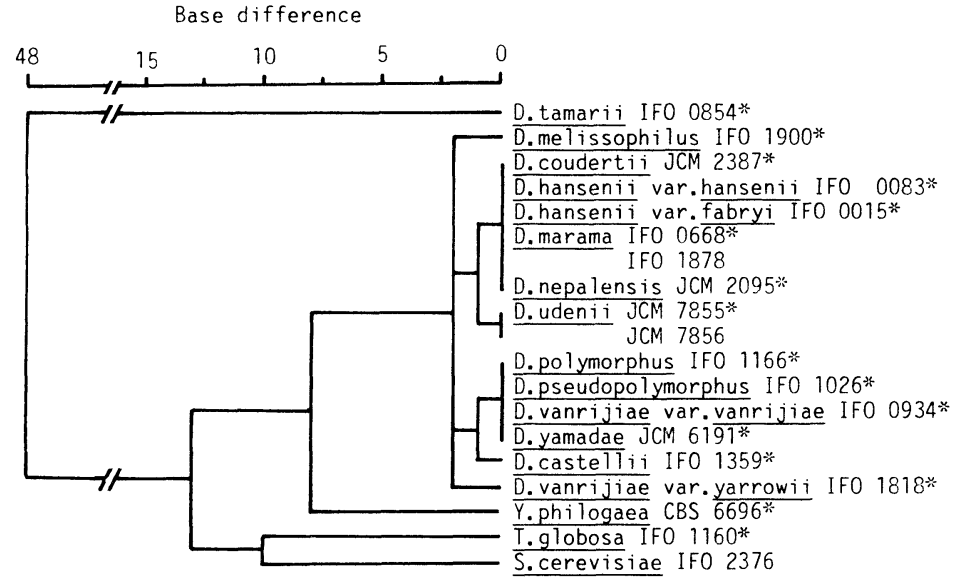

Fig. 6. A dendrogram based on the calculated number of base differences in the partial base sequences of positions 1611 through 1835 of 26S rRNA in strains of Debaryomyces, Torulaspora, and Yamadazyma species. The dendrogram was drawn by the simple linkage method (5). * Type strain.

separate cluster with 10 base differences. These two clusters were linked to each other at 13 base differences. The linkage of $D$. tamarii IFO 0854 was very distant (48 base differences).

Partial base sequences in positions 1451 through 1618 of $18 \mathrm{~S}$ rRNA

The primary partial base sequences of the strains of Debaryomyces, Torulaspora, and Yamadazyma species were aligned (Fig. 7). The base differences of $D$. 
hansenii var. hansenii IFO 0083 were at positions 1482 ( $\mathrm{G}$ to A), 1509 (U to $\mathrm{G}$ ), and 1512 (U to A), compared with S. cerevisiae IFO 2376. The finger print segment (19) (positions 1488 through 1491 in $S$. cerevisiae) was characterized by AUUAA ( 3 base differences with $S$. cerevisiae IFO 2376) in all the strains of Debaryomyces species. The base sequences of T. globosa IFO 1160 (CUAA) and Y. philogaea CBS 6696 (AUUAA) coincided in the finger print segment with those of $S$. cerevisiae IFO 2376 and $D$. hansenii var. hansenii IFO 0083, respectively. The number of base differences was calculated among the strains examined.

As shown in Fig. 8, the base difference numbered 1 to 0 : the strains of Debaryomyces species constituted a single group, which was divided into two subgroups. The first subgroup including the type species of the genus Debaryomyces was comprised of the strains of $D$. hansenii var. hansenii, $D$. hansenii var. fabryi, $D$. marama, $D$. coudertii, D. melissophilus, D. nepalensis, and D. udenii, and the second subgroup was comprised of the strains of $D$. castellii, $D$. polymorphus, D. pseudo-

1 D.castellii IF0 $1359 *$

2 D.coudertii JCM $2387 *$

3 D.hanseni var.hansenii IF0 $0083 *$

4 D.hansenii var.fabryi IF0 0015 *

5 D.marama IF0 0668*

$6 \quad$ IFO 1878

7 D.melissophilus IF0 1900 *

8 D.nepalensis JCM 2095*

9 D.polymorphus IF0 $1166 *$

10 D.pseudopolymorphus IF0 1026*

11 D.tamarii IF0 0854 *

12 D.udenii JCM 7855 *

13 JCM 7856

14 D.vanrijiae var.vanrijiae IF0 $0934 *$

15 D.vanrijiae var.yarrowii IF0 1818*

16 D.yamadae JCM $6191 *$

17 T.globosa IF0 $1160 *$

18 Y.philogaea CBS $6696 *$

19 S.cerevisiae IF0 2376

\section{$\begin{array}{llllllll}.1451 & .1461 & .1471 & .1481 & .1488 & .1492 & .1501 & .1511\end{array}$}

GGGCCGCACG CGCGCUACAC UGACGGAGCC AGCGAGU AUUAA.CCUUGGCCG AGAGGUCUGG GAAAUCUUGU GGGCCGCACG CGCGCUACAC UGACGGAGCC AACGAGU AUUAA.CCUUGGCCG AGAGGUCUGG GAAAUCUUGU GGGCCGCACG CGCGCUACAC UGACGGAGCC AACGAGU AUUAA.CCUUGGCCG AGAGGUCUGG GAAAUCUUGU GGGCCGCACG CGCGCUACAC UGACGGAGCC AACGAGU AUUAA.CCUUGGCCG AGAGGUCUGG GAAAUCUUGU GGGCCGCACG CGCGCIJACAC UGACGGAGCC AACGAGU AUUAA.CCUUGGCCG AGAGGUCUGG GAAAUCUUGU GGGCCGCACG CGCGCUACAC UGACGGAGCC AACGAGU AUUAA.CCUUGGCCG AGAGGUCUGG GAAAUCUUGU GGGCCGCACG CGCGCUACAC UGACGGAGCC AACGAGU AULAAA.CCUUGGCCG AGAGGUCUGG GAAAUCUUGU GGGCCGCACG CGCGCUACAC UGACGGAGCC AACGAGU AUUAA.CCUUGGCCG AGAGGUCUGG GAAAUCUUGU GGGCCGCACG CGCGCUACAC UGACGGAGCC AGCGAGU AUUAA.CCUUGGCCG AGAGGUCUGG GAAAUCUUGU GGGCCGCACG CGCGCUACAC UGACGGAGCC AGCGAGU AUUAA.CCUUGGCCG AGAGGUCUGG GAAAUCUUGU GGGCCGCACG CGCGCUACAC UGACGCAGCC AACGAGU ACAUAACCUUGUCCG AAAGGUCUGG GUAAUCUUGU GGGCCGCACG CGCGCUACAC UGACGGAGCC AACGAGU AUUAA.CCUUGGCCG AGAGGUCUGG GAAAUCUUGU GGGCCGCACG CGCGCUACAC UGACGGAGCC AACGAGU AUUAA.CCUUGGCCG AGAGGUCUGG GAAAUCUUGU GGGCCGCACG CGCGCUACAC UGACGGAGCC AGCGAGU AUUAA.CCUUGGCCG AGAGGUCUGG GAAAUCUUGU GGGCCGCACG CGCGCUACAC UGACGGAGCC AGCGAGU AUUAA.CCUUGGCCG AGAGGUCUGG GAAAUCUUGU GGGCCGCACG CGCGCUACAC UGACGGAGCC AGCGAGU AUUAA.CCUUGGCCG AGAGGUCUGG GAAAUCUUGU GGGCCGCACG CGCGCUACAC UGACGGAGCC AGCGAGU CUAA..CCUUGGCCG AGAGGUCUGG GUAAUCUUGU GGGCCGCACG CGCGCUACAC UGACGGAGCC AGCGAGU AUUAA.CCUUGGCCG AGAGGUCUGG GAAAUCUUGU GGGCCGCACG CGCGCUACAC UGACGGAGCC AGCGAGU CUAA..CCUUGGCCG AGAGGUCUUG GUAAUCUUGU

2 GAAACLCCGU CGUGCUGGGG AUAGAGCAUU GUAAUUUUG CUCUUCAACG AGGAAUUCCU AGUAAGCGCA AGUCAUCAGC UUGCGUUGAU UACGUCCC

3 GAAACUCCGU CGUGCUGGGG AUAGAGCAUU GUAAUUAUUG CUCUUCAACG AGGAAUUCCU AGUAAGCGCA AGUCAUCAGC UUGCGUUGAU UACGUCCC

4 GAAACUCCGU CGUGCUGGG AUAGAGCAUU GUAAUUAUUG CUCUUCAACG AGGAAUUCCU AGUAAGCGCA AGUCAUCAGC UUGCGUUGAU UACGUCCC

5 GAAACUCCGU CGUGCUGGG AUAGAGCAUU GUAAUUAUUG CUCUUCAACG AGGAAUUCCU AGUAAGCGCA AGUCAUCAGC UUGCGUUGAU UACGUCCC

6 GAAACUCCGU CGUGCUGGGG AUAGAGCAUU GUAAUUAUUG CUCUUCAACG AGGAAUUCCU AGUAAGCGCA AGUCAUCAGC UUGCGUUGAU UACGUCCC

7 GAAACUCCGU CGUGCUGGG AUAGAGCAUU GUAAUUAUUG CUCUUCAACG AGGAAUUCCU AGUAAGCGCA AGUCAUCAGC UUGCGUUGAU UACGUCCC

8 GAAACUCCGU CGUGCUGGGG AUAGAGCAUU GUAAUUAUUG CUCUUCAACG AGGAAUUCCU AGUAAGCGCA AGUCAUCAGC UUGCGUUGAU UACGUCCC

9 GAAACUCCGU CGUGCUGGGG AUAGAGCAUU GUAAUUAUUG CUCUUCAACG AGGAAUULCCU AGUAAGCGCA AGUCAUCAGC UUGCGUUGAU UACGUCCC

10 GAAACUCCGU CGUGCUGGG AUAGAGCAUU GUAAUUAUUG CUCUUCAACG AGGAAUUCCU AGUAAGCGCA AGUCAUCAGC UUGCGUUGAU UACGUCCC

11 UAAACUGCGU CGUGCUGGG ACAGAGCUUU GCAAUUUUUG CUCUCGAACG AGGAAUUCCU AGUAGGCGCA AGUCAUCA.C UUGCGUCGAU UACGUCCC

12 GAAACUCCGU CGUGCUGGG AUAGAGCAUU GUAAUUAUUG CUCUUCAACG AGGAAUUCCU AGUAAGCGCA AGLCAUCAGC UUGCGUUGAU UACGUCCC

13 GAAACUCCGU CGUGCUGGGG AUAGAGCAUU GUAAUUAUUG CUCUUCAACG AGGAAUUCCU AGUAAGCGCA AGUCAUCAGC UUGCGUUGAU UACGUCCC

14 GAAACUCCGU CGUGCUGGG AUAGAGCAUU GUAAUUAUUG CUCUUCAACG AGGAAUUCCU AGUAAGCGCA AGUCAUCAGC UUGCGUUGAU UACGUCCC

15 GAAACUCCGU CGUGCUGGG AUAGAGCAUU GUAAUUAUUG CUCUUCAACG AGGAAUUCCU AGUAAGCGCA AGUCAUCAGC UUGCGUUGAU UACGUCCC

16 GAAACUCCGU CGUGCUGGG AUAGAGCAUU GUAAUUAUUG CUCUUCAACG AGGAAUUCCU AGUAAGCGCA AGUCAUCAGC UUGCGUUGAU UACGUCCC

17 GAAACUCCGU CGUGCUGGG AUAGAGCAUU GUAAUUAUUG CUCUUCAACG AGGAAUUCCU AGUAAGCGCA AGUCAUCAGC UUGCGUUGAU UACGUCCC

18 GAAACUCCGU CGUGCUGGG ALAGAGCAUU GUAAUUAUUG CUCUUCAACG AGGAAUUCCU AGUAAGCGCA AGUCAUCAGC UUGCGUUGAU UACGUCCC

19 GAAACUCCGU CGUGCUGGG AUAGAGCAUU GUAAUUAUUG CUCUUCAACG AGGAAUUCCU AGUAAGCGCA AGUCAUCAGC UUGCGUUGAU UACGUCCC

Fig. 7. The primary partial base sequences in positions 1451 through 1618 of $18 \mathrm{~S}$ rRNA in strains of Debaryomyces, Torulaspora, and Yamadazyma species. The primary partial sequences were aligned. The arabic numerals indicate positions in $S$. cerevisiae (12). * Type strain. 


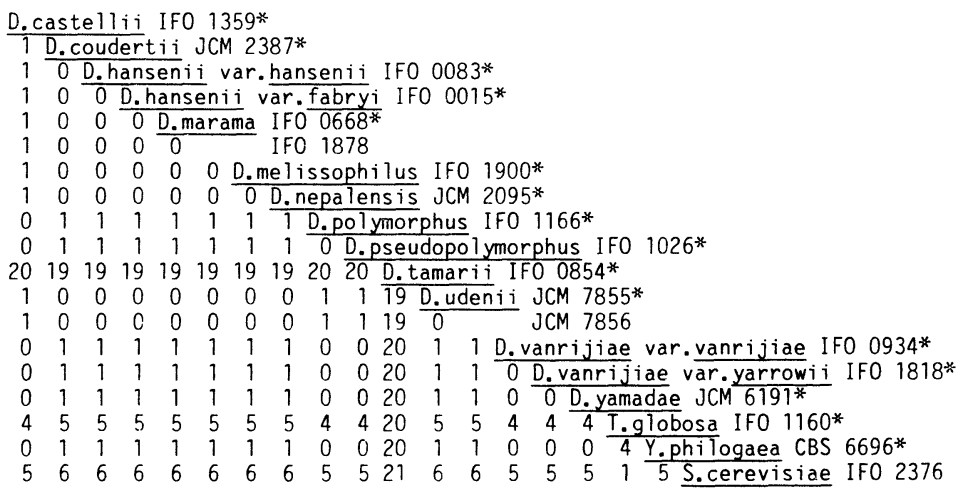

Fig. 8. A triangle matrix based on the calculated number of base differences in the partial base sequences of positions 1451 through 1618 of $18 \mathrm{~S}$ rRNA in strains of Debaryomyces, Torulaspora, and Yamadazyma species. The number of base differences was calculated in positions 1451 through 1618 (168 bases) of 18S rRNA. * Type strain.

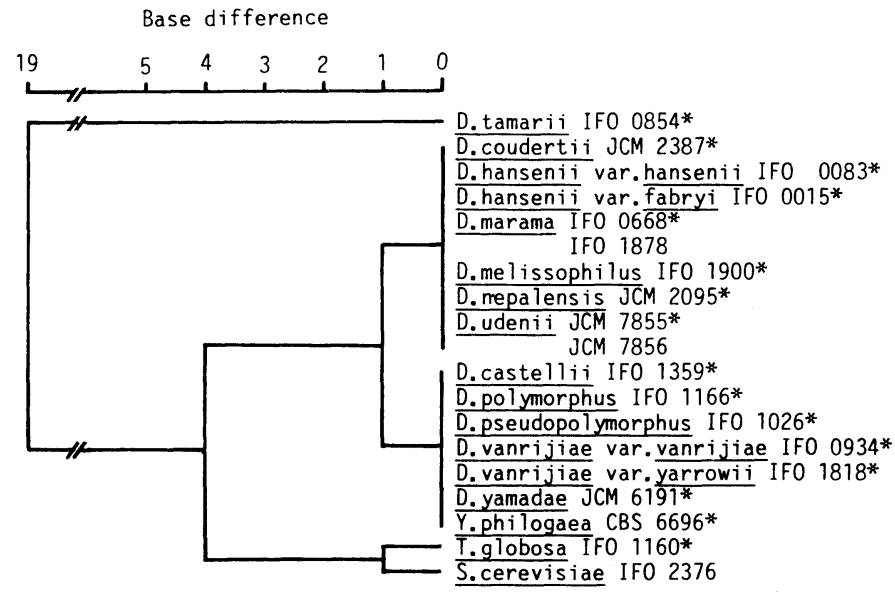

Fig. 9. A dendrogram based on the calculated number of base differences in the partial base sequences of positions 1451 through 1618 of $18 \mathrm{~S}$ rRNA in strains of Debaryomyces, Torulaspora, and Yamadazyma species. The dendrogram was drawn by the simple linkage method (5). * Type strain.

polymorphus, $D$. vanrijiae var. vanrijiae, $D$. vanrijiae var. yarrowii, and $D$. yamadae. Yamadazyma philogaea CBS 6696, the type strain of the type species of the genus Yamadazyma represented the same partial base sequence as the members of the second subgroup of the genus Debaryomyces. Torulaspora globosa IFO 1160 was very similar to $S$. cerevisiae IFO 2376 in the base sequence: it had 1 base difference. The base sequence of $D$. tamarii IFO 0854 was quite different (base differences, 19-20). 
Based on the calculated number of base differences, a dendrogram was drawn by the simple linkage method (5). As shown in Fig. 9, all the strains of Debaryomyces species were linked to each other at 1-0 base difference. The type strain of the type species of the genus Debaryomyces, D. hansenii var. hansenii IFO 0083 was included in the first subcluster (subgroup) of the genus Debaryomyces. Yamadazyma philogaea CBS 6696 was included in the second subcluster. Torulaspora globosa IFO 1160 was linked to $S$. cerevisiae IFO 2376 at 1 base difference, and then to the strains of Debaryomyces and Yamadazyma species at 4 base differences. Debaryomyces tamarii IFO 0854 was quite different, the linkage of which was at 19 base differences.

\section{DISCUSSION}

The type species, Debaryomyces globosus Klöcker, of the genus Debaryomyces Klöcker, was classed in the genus Torulaspora Lindner as $T$. globosa (Klöcker) van der Walt et Johannsen (16). In the present study, the accommodation of the species as T. globosa (16) and the proposal of the genus Debaryomyces Lodder et Kregervan Rij with the type species, D. hansenii (Zopf) Lodder et Kreger-van Rij (11) proved to be adequate phylogenetically. Phylogenetically, the $\mathrm{Q}_{6}$-equipped species, T. globosa was close to $S$. cerevisiae (Q-6) (1 base difference in partial base sequence of $18 \mathrm{~S}$ rRNA), rather than to $D$. hansenii (Q-9) $(13,20,22)$.

The members of the genus Debaryomyces Lodder et Kreger-van Rij constituted a single group (cluster) phylogenetically. All the species of the genus Debaryomyces had partial base sequences similar to each other except for D. tamarii. The maximum homologies were 79-99\% in the partial base sequence (positions 493 through 622,130 bases) of 26S rRNA, and the number of base differences was 10 in the partial base sequence (positions 1451 through 1618, 168 bases) of $18 \mathrm{~S}$ rRNA. Similar results were obtained in the partial base sequence in positions 1611 through 1835 (225 bases) of 26S rRNA (base differences, 5-0).

Kurtzman and Robnett (9) reported the partial base sequences of $18 \mathrm{~S}$ and $25 \mathrm{~S}$ (26S) rRNAs of the members of the genus Debaryomyces. They recognized three subclusters in the genus Debaryomyces Lodder et Kreger-van Rij except for $D$. tamarii. Our results coincided with theirs, however, they did not examine the strains of $D$. udenii. According to their sequence data of 18S rRNA (9), the same base sequences, AUUAA and CUAA (their positions 1467-1489), as ours for Debaryomyces species and $S$. cerevisiae, respectively, are read in the finger print segment which represents one of the genus-specific base sequences (19). Based on the partial base sequences in positions 1451 through 1618 (168 bases) of 18S rRNA, we divided the genus Debaryomyces into two subgroups (subclusters). Debaryomyces melissophilus constituted their third subcluster (9). In contrast, this species was placed in our first subgroup as $D$. hansenii, $D$. marama, and $D$. nepalensis were. However, our partial base sequencing of 26S rRNA gave a somewhat different situation to this species; the maximum homologies were slightly lower (79-86\%), 
and the base differences were slightly higher (5-2).

Kreger-van Rij (7) observed that the strain of D. tamarii does not produce ascospores. The present study has demonstrated that the species is not classified in the genus Debaryomyces.

Debaryomyces udenii was recently described as a new species of the genus Debaryomyces (18). This species is somewhat different morphologically from the presently accepted species of the genus Debaryomyces. It produces colliculate to pusticulate rather than verrucate ascospores. The results obtained have indicated that $D$. udenii was not included in the second subgroup (subcluster) as the physiologically related Debaryomyces species, D. castellii, D. pseudopolymorphus, and $D$. yamadae, but in the first subgroup as $D$. hansenii, the type species of the genus Debaryomyces, in the partial base sequence determinations of $18 \mathrm{~S}$ rRNA (positions 1451 through 1618, 168 bases).

It is notable that $Y$. philogaea, the type species of the $\mathrm{Q}_{9}$-equipped genus Yamadazyma Billon-Grand (3), had the same partial base sequence in 18S rRNA as the members in the second subgroup (or subcluster) of the genus Debaryomyces (Q-9) such as D. castellii, D. pseudopolymorphus, and D. yamadae. However, the number of base differences was not so small (10-8), compared with Debaryomyces species, in the partial base sequence (positions 1611 through 1835, 225 bases) of $26 \mathrm{~S}$ rRNA. The species of the genus Yamadazyma are characterized by producing hatshaped ascospores. The partial base sequences of $18 \mathrm{~S}$ and $26 \mathrm{~S}$ rRNAs of the remaining species of the genus Yamadazyma will be presented elsewhere.

We express our thanks to Dr. J. P. van der Walt, Pretoria, South Africa for his guidance and encouragement of this experiment. Thanks are also due to Dr. M. Suzuki and Dr. T. Nakase, Japan Collection of Microorganisms, Institute of Physical and Chemical Research, Wako, Saitama, Japan and to Dr. D. Yarrow, Centraalbureau voor Schimmelcultures, Delft, The Netherlands, for sending us yeast cultures. This work was supported by a Grant-in-Aid (No. 2806022) for Scientific Research from the Ministry of Education, Science and Culture of Japan.

\section{REFERENCES}

1) Billon-Grand, G., Coenzyme Q de quelques espèces du genre Pichia. Détermination qualitative et quantitative. Mycopathologia, 90, 101-106 (1985).

2) Billon-Grand, G., Minor ubiquinones of the yeast coenzyme $Q$ system: Importance in the taxonomy of the yeasts. J. Gen. Appl. Microbiol., 33, 381-390 (1987).

3 ) Billon-Grand, G., A new ascosporogenous yeast genus: Yamadazyma gen. nov. Mycotaxon, 35, 201-204 (1989).

4) Georgiev, O. I., Nikolaev, N., Hadjiolov, A. A., Skryabin, K. G., Zakharyev, V. M., and Bayev, A. A., The structure of the yeast ribosomal RNA genes. 4. Complete sequence of 25S rRNA gene from Saccharomyces cerevisiae. Nucleic Acids Res., 9, 6953-6958 (1981).

5) Kaneko, T., 7. Data kaiseki (7. Data analysis). In Biseibutsu no Kagaku Bunrui Jikkenho (Methods in Chemotaxonomy of Microorganisms) (in Japanese), ed. by Komagata, K., Japan Scientific Societies Press, Tokyo (1982), p. 309-346.

6) Kocková-Kratochvilová, A., Sláviková, E., and Jensen, V., Numerical taxonomy of the yeast genus Debaryomyces Lodder et Kreger-van Rij. J. Gen. Microbiol., 104, 257-268 (1978). 
7) Kreger-van Rij, N. J. W., Genus 7. Debaryomyces Lodder et Kreger-van Rij nom. cons. In The Yeasts: A Taxonomic Study, 3rd ed., ed. by Kreger-van Rij, N. J. W., Elsevier Science Publishers, Amsterdam (1984), p. 130-145.

8) Kreger-van Rij, N. J. W. and Veenhuis, M., Ultrastructure of the ascospores of some species of the Torulaspora group. Anionie van Leeuwenhoek: J. Microbiol. Serol., 42, 445-455 (1976).

9) Kurtzman, C. P. and Robnett, C. J., Phylogenetic relationships among species of Saccharomyces, Schizosaccharomyces, Debaryomyces, and Schwanniomyces determined from partial ribosomal RNA sequences. Yeast, 7, 61-72 (1991).

10) Lane, D. J., Pace, B., Olsen, G. J., Stahl, D. A., Sogin, M. L., and Pace, N. R., Rapid determination of $16 \mathrm{~S}$ ribosomal RNA sequences for phylogenetic analyses. Proc. Natl. Acad. Sci. U.S.A., 82, 6955-6959 (1985).

11) Lodder, J. and Kreger-van Rij, N. J. W., Proposal (446) for the conservation of the generic name Debaryomyces Lodder et Kreger-van Rij against Debaryomyces Klöcker. Taxon, 27, 306-307 (1978).

12) Mankin, A. S., Skryabin, K. G., and Rubtsov, P. M., Identification of ten additional nucleotides in the primary structure of yeast 18S rRNA. Gene, 44, 143-145 (1986).

13) Nakase, T. and Suzuki, M., Taxonomic studies on Debaryomyces hansenii (Zopf) Lodder et Kreger-van Rij and related species. I. Chemotaxonomic investigations. J. Gen. Appl. Microbiol., 31, 49-69 (1985).

14) Nakase, T. and Suzuki, M., Taxonomic studies on Debaryomyces hansenii (Zopf) Lodder et Kreger-van Rij and related species. II. Practical discrimination and nomenclature. J. Gen. Appl. Microbiol., 31, 71-86 (1985).

15 ) Price, C. W., Fuson, G. B., and Phaff, H. J., Genome comparison in yeast systematics: Delimitation of species within the genera Schwanniomyces, Saccharomyces, Debaryomyces, and Pichia. Microbiol. Rev.. 42, 161-193 (1978).

16) van der Walt, J. P. and Johannsen, E., The genus Torulaspora Lindner. C.S.I.R. Res. Rep., 325, 1-23 (1975).

17) van der Walt, J. P. and Johannsen, E., Debaryozyma yamadae spec. nov. (Saccharomycetaceae). J. Gen. Appl. Microbiol., 26, 217--222 (1980).

18) van der Walt, J. P., Smith, M. Th., and Yamada, Y., Debaryomyces udenii sp. nov. (Saccharomycetaceae), a new species from soil. Antonie van Leeuwenhoek: J. Gen. Mol. Microbiol., 56, 233-239 (1989).

19) Yamada, Y. and Kawasaki, H., The molecular phylogeny of the $\mathrm{Q}_{8}$-equipped basidiomycetous yeast genera Mrakia Yamada et Komagata and Cystofilobasidium Oberwinkler et Bandoni based on the partial sequences of $18 \mathrm{~S}$ and $26 \mathrm{~S}$ ribosomal ribonucleic acids. J. Gen. Appl. Microbiol., 35, 173-183 (1989).

20) Yamada, Y. and Kondo, K., Taxonomic significance of the coenzyme Q system in yeasts and yeast-like fungi (2). In Fermentation Technology Today: Proc. 4th Int. Ferment. Symp., Kyoto 1972, ed. by Terui, G., Society of Fermentation Technology, Japan, Osaka (1972), p. 781-784.

21) Yamada, Y., Nagahama, T., Banno, I., Giménez-Jurado, G., and van Uden, N., The phylogenetic relationship of Kurtzmanomyces tardus Giménez-Jurado et van Uden (Cryptococcaceae) based on the partial sequences of $18 \mathrm{~S}$ and $26 \mathrm{~S}$ ribosomal RNAs. J. Gen. Appl. Microbiol., 37, 321-324 (1991).

22) Yamada, Y., Nojiri, M., Matsuyama, M., and Kondo, K., Coenzyme Q system in the classification of the ascosporogenous yeast genera Debaryomyces, Saccharomyces, Kluyveromyces, and Endomycopsis. J. Gen. Appl. Microbiol., 22, 325-337 (1976).

23) Yamada, Y., Okada, T., Ueshima, O., and Kondo, K., Coenzyme Q system in the classification of the ascosporogenous yeast genera Hansenula and Pichia. J. Gen. Appl. Microbiol., 19, 189-208 (1973). 